\title{
Long noncoding RNA colon cancer associated transcript-1 promotes the proliferation, migration and invasion of cervical cancer
}

\author{
LIGANG JIA $^{1,2}$, YUAN ZHANG ${ }^{2}$, FEI TIAN ${ }^{2}$, ZHAOPING CHU ${ }^{2}$ and HONG XIN ${ }^{3}$ \\ ${ }^{1}$ Department of Gynecology, The Second Hospital of Hebei Medical University; \\ ${ }^{2}$ Department of Gynecology, Hebei General Hospital; ${ }^{3}$ Department of Obstetrics, The Second \\ Hospital of Hebei Medical University, Shijiazhuang, Hebei 050000, P.R. China
}

Received April 7, 2016; Accepted May 8, 2017

DOI: $10.3892 / \mathrm{mmr} .2017 .7302$

\begin{abstract}
Previous studies have revealed significant roles for long noncoding RNA (lncRNA) in the tumorigenesis, metastasis and invasion of various tumors, including cervical cancer. The present study aimed to investigate the potential roles of lncRNA colon cancer associated transcript 1 (CCAT1) in the metastasis and invasion of cervical cancer, and to reveal the potential underlying mechanism. The mRNA expression of lncRNA CCAT1 in cervical cancer tissue was measured using the reverse transcription-quantitative polymerase chain reaction, and the association between lncRNA CCAT1 and the metastasis of cervical cancer was analyzed. The effects of lncRNA CCAT1 expression on HeLa cell viability, and migration and invasion were also analyzed by MTT and Transwell assays. The results demonstrated that lncRNA CCAT1 was highly expressed in the cervical cancer tissue compared with the adjacent normal tissue. High expression of lncRNA CCAT1 was positively associated with tumor size, and there was correlation between high lncRNA CCAT1 expression and a poor survival rate of cervical cancer. The cell viability, and migratory and invasive abilities were suppressed by silencing CCAT1. The results of the present study indicate that lncRNA CCAT1 was highly expressed in cervical cancer, and may serve important roles in promoting the progression and metastasis of cervical cancer.
\end{abstract}

\section{Introduction}

Cervical cancer remains one of the most common female malignancies worldwide (1), and is characterized by

Correspondence to: Dr Hong Xin, Department of Obstetrics, The Second Hospital of Hebei Medical University, 215 Heping West Road, Shijiazhuang, Hebei 050000, P.R. China

E-mail: xinhong56620@126.com

Key words: cervical cancer, long noncoding RNA, colon cancer associated transcript 1 , cell proliferation, cell migration, cell invasion migration and invasion (2), resulting in a poor 5-year survival rate (3). Therefore, the development of novel methods for the improvement of cervical cancer treatment and prognosis is vital for improving the diagnosis and treatment of cervical cancer.

Long noncoding RNAs (lncRNAs; >200 nucleotides) have been demonstrated to exhibit crucial roles in tumorigenesis, migration and invasion in recent years $(4,5)$. For example, the upregulated lncRNA HOX transcript antisense RNA (HOTAIR) promotes the progression of hepatocellular cancer (6), and IncRNA H19 promotes the tumorigenesis and metastasis of gastric cancer (7). Generally, the underlying mechanism of IncRNA gene expression regulation may be at the transcriptional or post-transcriptional level (8). To date, several lncRNAs, including focally amplified lncRNA in epithelial cancer (FAL1), HOTAIR, metastasis-associated lung adenocarcinoma transcript-1 and cervical carcinoma expressed proliferating cell nuclear antigen regulatory lncRNA, have been reported to serve pivotal roles in the progression or metastasis of cervical cancer (9-12). lncRNA colon cancer associated transcript 1 (CCAT1) is highly expressed in colon cancer tissue, and has been reported to be useful in the detection of colon cancer and tumor-associated tissues (13). The roles of lncRNA CCAT1 in the biology of tumors has only been reported in a number of tumor types $(14,15)$. The proliferation and invasion of colon cancer cells was promoted by the c-myc-mediated activation of lncRNA CCAT1 (16). Ma et al (17) demonstrated that IncRNA CCAT1 promotes the development of gallbladder cancer by negatively modulating the expression of miR-218-5p. These previous studies indicate that lncRNA CCAT1 may function as an oncogene in tumors. However, few studies have reported the association between lncRNA CCAT1 expression, and the progression and development of cervical cancer.

The present study investigated the expression of lncRNA CCAT1 in cervical cancer tissue, and analyzed the association between lncRNA CCAT1 expression and HeLa cell viability, metastasis and invasion. The present study aimed to investigate the potential roles of lncRNA CCAT1 in the metastasis of cervical cancer and to identify the potential underlying mechanism. 


\section{Materials and methods}

Patients and samples. A total of 30 patients aged 23-62 years who were diagnosed with cervical cancer at Hebei Provincial People's Hospital (Shijiazhuang, China) between June 2014 and December 2015 were enrolled in the present study. Informed consent was obtained from all patients and all procedures were approved by the Protection of Human Ethics Committee of the Hebei Provincial People's Hospital. The cervical cancer diagnosis was pathologically confirmed, and cancer tissues and adjacent normal tissue were obtained from clinically ongoing surgical specimens with informed consent. Tissues size was measured and all of the samples were snap-frozen with liquid nitrogen and stored at $-80^{\circ} \mathrm{C}$ until RNA extraction.

Cell culture and transfection. HeLa human cervical cancer cells (European Collection of Authenticated Cell Cultures; Public Health England; Salisbury, UK) were cultured in Dulbecco's modified Eagle's medium (DMEM; Gibco; Thermo Fisher Scientific, Inc., Waltham, MA, USA) supplemented with $10 \%$ fetal bovine serum (FBS; Thermo Fisher Scientific, Inc.) at $37^{\circ} \mathrm{C}$ in an atmosphere containing $5 \% \mathrm{CO}_{2}$. The full-length of CCAT1 encoding sequences were amplified by using the PfuUltra II fusion HS DNA Polymerase (Agilent Technologies, Inc., Santa Clara, CA, USA). The thermocycling conditions were: $95^{\circ} \mathrm{C} 45 \mathrm{sec}, 60^{\circ} \mathrm{C} 15 \mathrm{sec}, 95^{\circ} \mathrm{C} 30 \mathrm{sec}, 30$ cycles. Then CCAT1 sequences were cloned into pcDNA3.1 vector (Invitrogen; Thermo Fisher Scientific, Inc.) as pcDNA3.1-CCAT1. The experimental procedures were performed as previously described (18). Primers used for CCAT1 amplification were 5'-CTAGCTAGCACAACATCGACTTTGAAGTT-3' (forward) and 5'-CCCAAGCTTAAGACTTAATATACTTATATTT A-3' (reverse). The small interfering (si)RNA for CCAT1 was designed and synthesized by RiboBio (Guangzhou RiboBio Co., Ltd., Guangzhou, China), and the sequence was as follows: CCAT1 siRNA (si-CCAT1: 5'-CAUUAACCUGCUAUCCUC UUUACAA-3'). Si-CCAT1 was transfected into HeLa cells for $48 \mathrm{~h}$ by using the Lipofectamine $2000^{\circledR}$ (Invitrogen; Thermo Fisher Scientific, Inc.), according to the manufacturer's instructions. Cells without transfections were used as a negative control.

MTT assay. Effects of 1ncRNA CCAT1 expression on cell proliferation were assessed using MTT assay as previously described (19). Cells cultured in DMEM medium containing $10 \%$ FBS at logarithmic stage $\left(5 \times 10^{3}\right)$ were transfected in 96-well plates. After $24 \mathrm{~h}$ cultivation, supernatant was removed and MTT $(20 \mu \mathrm{l})$ was added every $24 \mathrm{~h}$ and incubated for $4 \mathrm{~h}$. Subsequently, $150 \mu 1$ dimethyl sulfoxide was added to the cells for $10 \mathrm{~min}$. Absorbance of cells in each well was observed at $570 \mathrm{~nm}$ using an absorption spectrophotometer (Olympus Corporation, Tokyo, Japan).

Colony assay. HeLa cells were seeded onto $10 \mathrm{~cm}$ plates at a density of $1 \times 10^{3}$ cells/well. Cells were treated and allowed to grow for 14 days until visible clones appeared. Cell colony formation was detected by staining with $0.1 \%$ crystal violet solution, air dried and counted.

Cell migration and invasionassays. Cell migration and invasion assays were performed using Transwell migration chambers
( $8 \mu \mathrm{m}$ pore size). The membranes for the invasion assay were coated with a diluted extracellular matrix (Matrigel, consisting of the laminin and type IV collagen) solution (Sigma-Aldrich; Merck KGaA, Darmstadt, Germany). Cells were seeded in the upper chamber at a density of $5 \times 10^{4}$ cells/well with serum-free medium following transfection. Medium containing $10 \%$ FBS, served as a chemoattractant in the lower chamber. After $24 \mathrm{~h}$ incubation at $37^{\circ} \mathrm{C}$, non-invasive/migratory cells on the top of the membrane were scraped and removed by cotton swabs, and the invasive/migratory cells were fixed in $4 \%$ methanol (NIST, USA) for $30 \mathrm{~min}$ at room temperature. Then $0.1 \%$ crystal violet (Sigma-Aldrich; Merck KGaA, Darmstadt, Germany) was used to stain for $20 \mathrm{~min}$ at room temperature and subsequently five randomly selected fields of view were counted using light microscopy at 400x magnification.

Reverse transcription-quantitative polymerase chain reaction (RT-qPCR). Total RNA from cervical cancer tissue was extracted using TRIzol ${ }^{\circledast}$ reagent (Invitrogen; Thermo Fisher Scientific, Inc.), followed by treatment with RQ1 RNase-free DNase I (Promega Corporation, Madison, WI, USA). Purified RNA mixed with nuclease-free water was used for cDNA synthesis using the reverse transcriptase PrimerScript 1st Strand cDNA Synthesis kit (Invitrogen; Thermo Fisher Scientific, Inc.). Expression was detected in an Eppendorf Mastercycler using the SYBR ExScript RT-PCR kit (Takara Biotechnology, Co., Ltd., Dalian, China) according to the manufacturer's protocol. The reaction procedure was as follows: $95^{\circ} \mathrm{C} 45 \mathrm{sec}, 95^{\circ} \mathrm{C} 15 \mathrm{sec}, 60^{\circ} \mathrm{C} 15 \mathrm{sec}, 45$ cycles. All the tests were repeated 3 times, and the relative quantitative analysis by $2^{-\Delta \Delta \mathrm{Ct}}$ method for the determination of LncRNA CCAT1 changes (20). Primers used were: LncRNA CCA T1, 5'-TTTATGCTTGAGCCTTGA-3' (forward) and 5'-CTT GCCTGAAATACTTGC-3' (reverse); internal GAPDH primer: 5'-TGTTGCCATCAATGACCCCTT-3' (forward) and 5'-CTCCACGACGTACTCAGCG-3' (reverse). GAPDH was selected as the internal control.

Statistical analysis. All experiments were repeated three times independently. Data are presented as the mean \pm standard deviation. Statistical analysis was performed using SPSS 19.0 software (IBM SPSS, Armonk, NY, USA). Significant differences were calculated using one-way analysis of variance followed by Dunnett's test. The correlation analysis was performed by using log rank to compare survival curves. $\mathrm{P}<0.05$ was considered to indicate a statistically significant difference.

\section{Results}

Expression of IncRNA CCAT1 in cervical cancer tissue. The present study analyzed the relative mRNA expression of IncRNA CCAT1 in cervical cancer tissue by RT-qPCR analysis. The results demonstrated that lncRNA CCAT1 exhibited increased expression in the cervical cancer tissue compared with the adjacent tissue $(\mathrm{P}<0.05$; Fig. 1A). Consequently, the association between relative mRNA expression of lncRNA CCAT1 in tumor tissue and tumor size was also analyzed, and the results demonstrated that as the expression of lncRNA CCAT1 increased the tumor size increased (Fig. 1B). In 
A

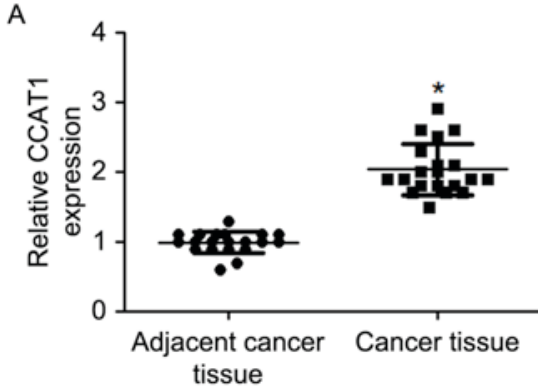

B

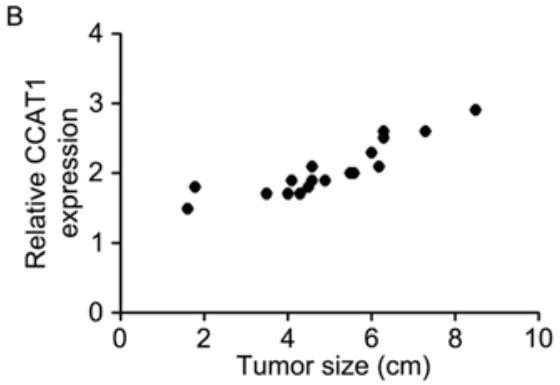

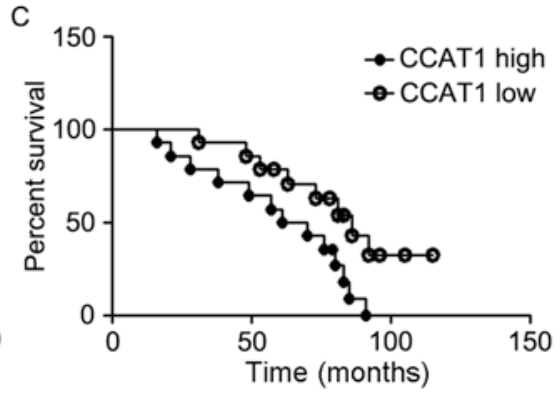

Figure 1. Expression of IncRNA CCAT1 in cervical cancer tissue. (A) IncRNA CCAT1 was highly expressed in cervical cancer tissue compared with adjacent normal tissue. "P<0.05 vs. adjacent normal tissue. (B) lncRNA CCAT1 expression was positively associated with tumor size in cervical cancer. (C) High IncRNA CCAT1 expression was associated with a poor survival rate in cervical cancer. IncRNA, long noncoding RNA; CCAT1, colon cancer associated transcript 1 .

A

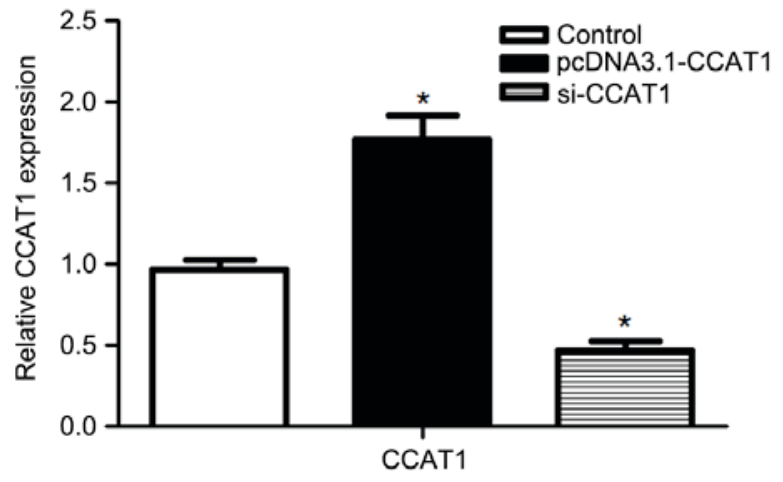

C

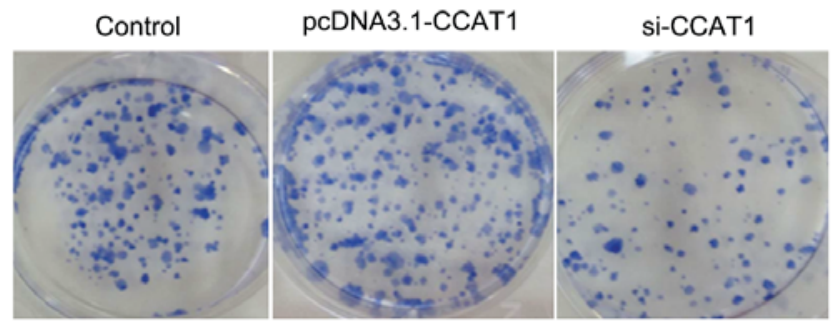

B

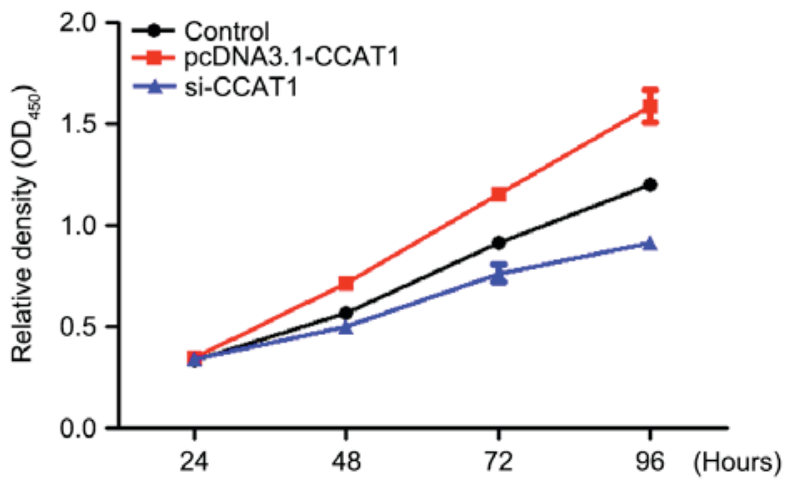

D

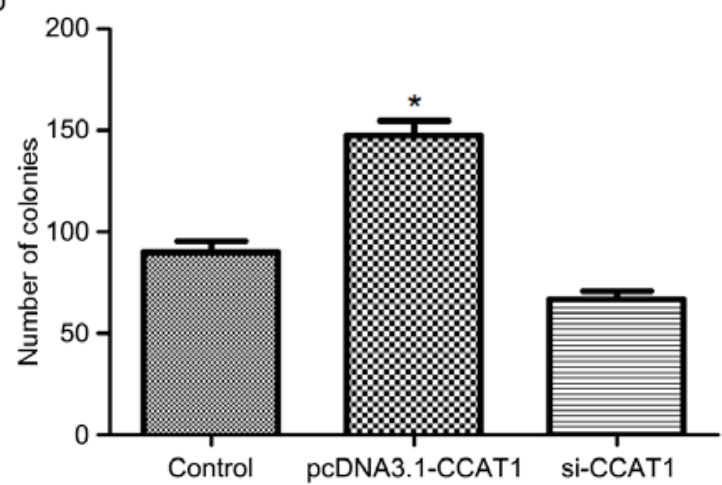

Figure 2. IncRNA CCAT1 promotes the proliferation of HeLa cells. (A) lncRNA CCAT1 expression was increased in cells transfected with the overexpression plasmid and reduced in cells transfected with si-CCAT1, compared with control cells. (B) lncRNA CCAT1 overexpression increased cell viability and silencing of lncRNA CCAT1 reduced cell viability compared with control cells. (C) HeLa cell colonies were stained with $0.1 \%$ crystal violet. (D) Number of HeLa cell colonies counted by crystal violet staining. The number of colonies was significantly increased by overexpression of lncRNA CCAT1 and significantly reduced by silencing lncRNA CCAT1. "P<0.05 vs. control. IncRNA, long noncoding RNA; CCAT1, colon cancer associated transcript 1; si-CCAT1, small interfering RNA against CCAT1; OD, optical density.

addition, the correlation between survival and lncRNA CCAT1 expression was also investigated, and the results demonstrated that high IncRNA CCAT1 expression was associated with a decreased survival rate compared with low CCAT1 expression (Fig. 1C). The results may indicate that the expression of 1ncRNA CCAT1 is positively associated with the tumor size, and associated with a poor survival rate in patients with cervical cancer.

Effects of IncRNA CCAT1 expression on HeLa cell viability in vitro. IncRNA CCAT1 was overexpressed and silenced in
HeLa cells (Fig. 2). Expression of lncRNA CCAT1 was significantly increased following pcDNA3.1-CCAT1 transfection compared with the control, while it was significantly reduced by transfection with si-CCAT1 transfection compared with the control $(\mathrm{P}<0.05$; Fig $2 \mathrm{~A})$. Subsequently, the viability of HeLa cells was analyzed, and results demonstrated that cell viability was increased by 1ncRNA CCAT1 overexpression, while it was reduced by silencing CCAT1 expression compared with control cells (Fig. 2B), indicating a positive association between lncRNA CCAT1 expression and HeLa cell viability. In addition, the colony assay also revealed that IncRNA 

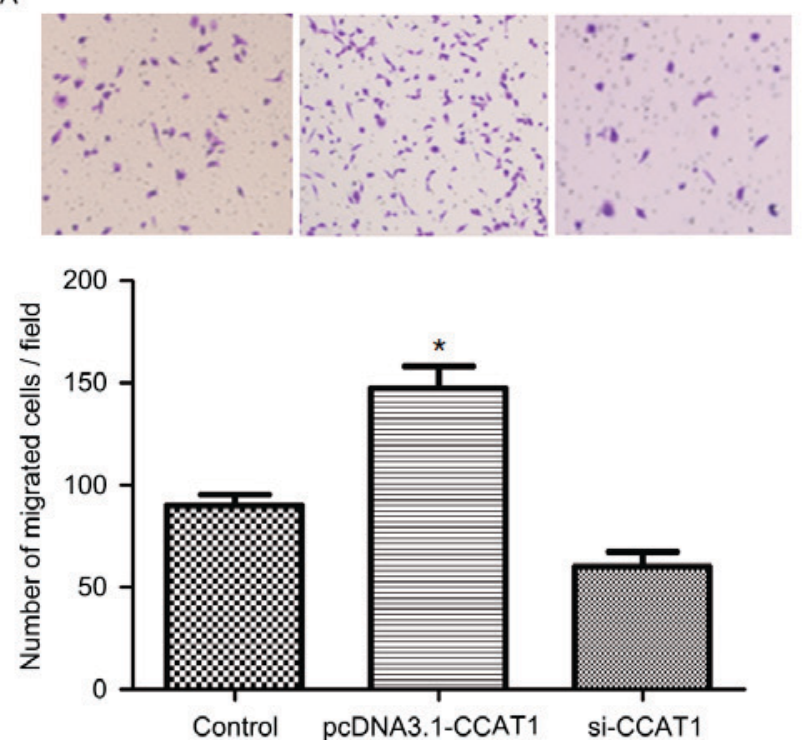
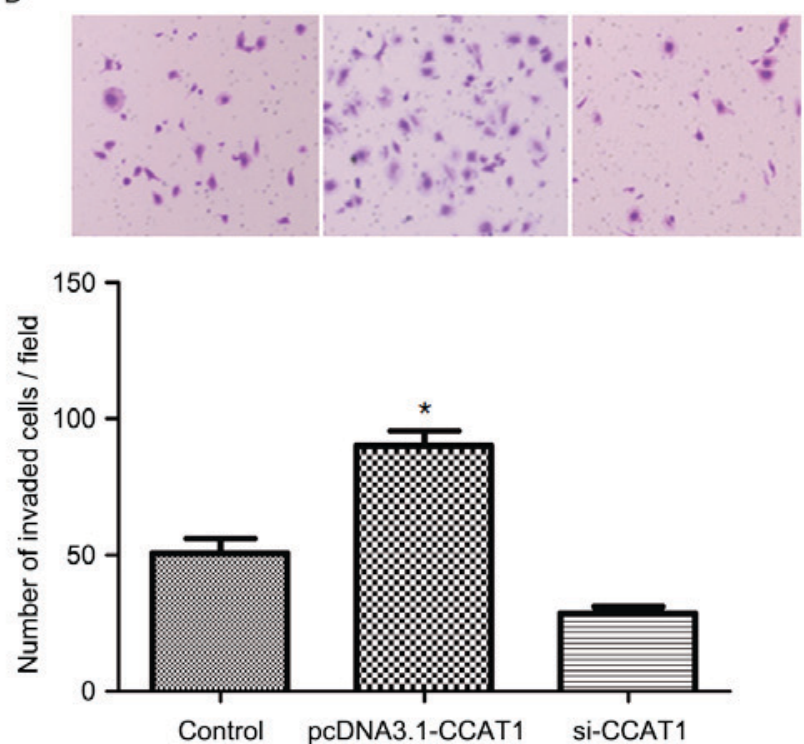

Figure 3. Effects of IncRNA CCAT1 expression on the migration and invasion of HeLa cells. (A) The number of migratory cells was significantly increased by lncRNA CCAT1 overexpression and significantly reduced by silencing CCAT1 compared with control cells. (B) The number of invasive cells was significantly increased by lncRNA CCAT1 overexpression and significantly reduced by silencing CCAT1. "P<0.05 vs. control. lncRNA, long noncoding RNA; CCAT1, colon cancer associated transcript 1; si-CCAT1, small interfering RNA against CCAT1.

CCAT1 overexpression increased the number of HeLa cell colonies compared with the control (Fig. 2C and D).

IncRNA CCAT1 overexpression promotes migration and invasion in HeLa cells. The influence of IncRNA CCAT1 expression on HeLa cell migration (Fig. 3A) and invasion (Fig. 3B) was investigated. The number of migratory or invasive cells was significantly increased by CCAT1 overexpression compared with the control group $(\mathrm{P}<0.05)$, while the number of migratory or invasive cells was decreased by silencing CCAT1, indicating that IncRNA CCAT1 expression was positively associated with cell migration and invasion in HeLa cells.

\section{Discussion}

Increasing evidence has demonstrated that lncRNAs serve pivotal roles in the biology of various tumors, including cervical cancer (21). Previous studies have demonstrated that lncRNA CCAT1 is abundantly expressed in several types of cancer, including colon and gastric cancer $(14,15)$. The present study analyzed the expression of 1ncRNA CCAT1 in cervical cancer tissue, and investigated the potential roles of lncRNA CCAT1 in the development and metastasis of cervical cancer. The results of the present study revealed that lncRNA CCAT1 was highly expressed in cervical cancer tissue and that its expression was positively associated with tumor size, indicating that the abnormal expression of lncRNA CCAT1 may be associated with cervical cancer.

In addition, the present study investigated the influence of 1ncRNA CCAT1 expression on the proliferation of HeLa cells and the results demonstrated that silencing lncRNA CCAT1 reduced the viability of HeLa cells compared with control HeLa cells. The roles of IncRNA CCAT1 in cervical cancer have not, to the best of our knowledge, previously been completely investigated. However, previous studies have demonstrated that colon cancer cell proliferation is promoted by the overexpression of 1ncRNA CCAT1 $(22,23)$. Similarly, Wang et al (24) demonstrated that upregulation of lncRNA CCAT1 promoted cell proliferation in esophageal squamous carcinoma and Liang et al (25) reported that lncRNA CCAT1 promoted the progression of hepatocellular carcinoma by a complex mechanism. Based on the results of the current study, we hypothesize that lncRNA CCAT1 upregulation may enhance cell proliferation in cervical cancer.

The present study also examined the effects of abnormal lncRNA CCAT1 expression on the migration and invasion of HeLa cells. Cell migration and invasion are involved in the metastasis of tumors (26). IncRNA CCAT1 was previously demonstrated to have crucial roles in the proliferation and invasion in various types of tumor, including colon cancer and hepatocellular cancer $(22,27)$. Additionally, previous studies have revealed that several lncRNAs serve roles in the invasion and migration of cervical cancer cells, including FAL1, MALAT1 and LET IncRNAs $(10,28,29)$. The results of the present study revealed that HeLa cell migration and invasion was suppressed by silencing lncRNA CCAT1, indicating that abnormal expression of 1ncRNA CCAT1 may be associated with cervical cancer migration and invasion.

In conclusion, the results of the present study revealed that lncRNA CCAT1 was upregulated in cervical cancer tissues, and that the abnormal expression of lncRNA CCAT1 may increase the progression and metastasis of cervical cancer by affecting cell proliferation, migration and invasion. The present study may provide a theoretical basis for the potential role of 1ncRNA CCAT1 as an oncogene in cervical cancer. Further studies are required to investigate the signaling pathways underlying the effects of abnormal lncRNA CCAT1 expression on cervical cancer in depth. 


\section{References}

1. Peng P, Huang L, Wang Y, You C, Cao W, Song H, Tan H and $\mathrm{Wu}$ Y: Effect of recombinant trichosanthin on proliferation of human cevical cancer Caski cells. Zhongguo Zhong Yao Za Zhi 36: 2539-2542, 2011 (In Chinese).

2. Ren CX, Zhao M, Na XU, Song YQ, Chen YP, Lv B and Yang G: Cancer-associated fibroblasts promotes migration and invasion of cervical epithelial cancer cells through IL-6-induced 'epithelial-mesenchymal' transition. Chin Oncol 24: 252-257, 2014.

3. Cao Z, Bao M, Miele L, Sarkar FH, Wang Z and Zhou Q: Tumour vasculogenic mimicry is associated with poor prognosis of human cancer patients: A systemic review and meta-analysis. Eur J Cancer 49: 3914-3923, 2013.

4. Wright CM: Chapter 5-Long Noncoding RNAs and Cancer. Epigenetic Cancer Therapy: 91-114, 2015.

5. Sahu A, Singhal U and Chinnaiyan AM: Long noncoding RNAs in Cancer: From function to translation. Trends Cancer 1: 93-109, 2015.

6. Bhan A and Mandal SS: LncRNA HOTAIR: A master regulator of chromatin dynamics and cancer. Biochim Biophys Acta 1856: 151-164, 2015.

7. Li H, Yu B, Li J, Su L, Yan M, Zhu Z and Liu B: Overexpression of lncRNA H19 enhances carcinogenesis and metastasis of gastric cancer. Oncotarget 5: 2318-2329, 2014.

8. Fahling M: Regulating gene expression at the post-transcriptional level: Spotlight on RNA-binding proteins. Curr Protein Pept Sci 13: 281-283, 2012

9. Lu H, Yu H, Lin L, Qi Z, Ma L, Li L and Su Y: Long non-coding RNA MALAT1 modulates radiosensitivity of HR-HPV+ cervical cancer via sponging miR-145. Tumor Biol 37: 1683-1691, 2016.

10. Hui H, Wu L, Fu Y, Zhou Y, Dong N and Hu Y: The effect of lncRNA FAL1 on cervical cancer cells proliferation, invasion and migration. J Mod Oncol: 2096-2099, 2015.

11. Yang M, Zhai X, Xia B, Wang Y and Lou G: Long noncoding RNA CCHE1 promotes cervical cancer cell proliferation via upregulating PCNA. Tumor Biol 36: 7615-7622, 2015.

12. Jing L, Yuan W, Ruofan D, Jinjin Y and Haifeng Q: HOTAIR enhanced aggressive biological behaviors and induced radio-resistance via inhibiting p21 in cervical cancer. Tumor Biol 36: 3611-3619, 2015.

13. Nissan A, Stojadinovic A, Mitrani-Rosenbaum S, Halle D, Grinbaum R, Roistacher M, Bochem A, Dayanc BE, Ritter G, Gomceli I, et al: Colon cancer associated transcript-1: A novel RNA expressed in malignant and pre-malignant human tissues. Int J Cancer 130: 1598-1606, 2012.

14. Kam Y, Rubinstein A, Naik S, Djavsarov I, Halle D, Ariel I, Gure AO, Stojadinovic A, Pan H, Tsivin V, et al: Detection of a long non-coding RNA (CCAT1) in living cells and human adenocarcinoma of colon tissues using FIT-PNA molecular beacons. Cancer Lett 352: 90-96, 2014.
15. Wang C, Hua L, Yao K, Chen J, Zhang J and Hu J: Long non-coding RNA CCAT2 is up-regulated in gastric cancer and associated with poor prognosis. Int J Clin Exp Pathol 8: 779-785, 2015.

16. He X, Tan X, Wang X, Jin H, Liu L, Ma L, Yu H and Fan Z: C-Myc-activated long noncoding RNA CCAT1 promotes colon cancer cell proliferation and invasion. Tumor Biol 35: 12181-12188, 2014.

17. Ma MZ, Chu BF, Zhang Y, Weng MZ, Qin YY, Gong W and Quan ZW: Long non-coding RNA CCAT1 promotes gallbladder cancer development via negative modulation of miRNA-218-5p. Cell Death Dis 6: e1583, 2015.

18. Wang Q, Zhang W and Hao S: LncRNA CCAT1 modulates the sensitivity of paclitaxel in nasopharynx cancers cells via miR-181a/CPEB2 axis. Cell Cycle 16: 795-801, 2017.

19. Moradhaseli S, Zare Mirakabadi A, Sarzaeem A, Kamalzadeh M and Haji Hosseini R: Cytotoxicity of ICD-85 NPs on human cervical carcinoma HeLa cells through caspase- 8 mediated pathway. Iran J Pharm Res 12: 155-163, 2013.

20. Livak KJ and Schmittgen TD: Analysis of relative gene expression data using real-time quantitative PCR and the 2(-Delta Delta C (T)) method. Methods 25: 402-408, 2001.

21. Peng L, Yuan X, Jiang B, Tang Z and Li GC: LncRNAs: Key players and novel insights into cervical cancer. Tumor Biol 37: 2779-2788, 2016

22. Marbacher S, Neuschmelting V, Andereggen L, Widmer HR, von Gunten M, Takala J, Jakob SM and Fandino J: Early brain injury linearly correlates with reduction in cerebral perfusion pressure during the hyperacute phase of subarachnoid hemorrhage. Intensive Care Med Exp 2: 30, 2014.

23. Xiang J, Yin Q, Chen T, Zhang Y, Zhang XO, Wu Z, Zhang S, Wang HB, Ge J, Lu X, et al: Human colorectal cancer-specific CCAT1-L lncRNA regulates long-range chromatin interactions at the MYC locus. Cell Res 24: 513-531, 2014

24. Wang CM, Wu QQ, Li SQ, Chen FJ, Tuo L, Xie HW, Tong YS, Ji L, Zhou GZ, Cao G, et al: Upregulation of the long non-coding RNA PlncRNA-1 promotes esophageal squamous carcinoma cell proliferation and correlates with advanced clinical stage. Dig Dis Sci 59: 591-597, 2013

25. Liang D, Yang SB, Xu FF and Zhang JH: Long noncoding RNA CCAT1 promotes hepatocellular carcinoma progression by functioning as let-7 sponge. J Exp Clin Cancer Res 34: 18, 2015.

26. Heerboth S, Housman G, Leary M, Longacre M, Byler S, Lapinska K, Willbanks A and Sarkar S: EMT and tumor metastasis. Clin Transl Med 4: 6, 2015.

27. Zhu H, Zhou X, Chang H, Li H, Liu F, Ma C and Lu J: CCAT1 promotes hepatocellular carcinoma cell proliferation and invasion. Int J Clin Exp Pathol 8: 5427-5434, 2015.

28. Jiang S, Wang H and Yang J: Low expression of long non-coding RNA LET inhibits carcinogenesis of cervical cancer. Int J Clin Exp Pathol 8: 806-811, 2015.

29. Yang L, Bai HS, Deng Y and Fan L: High MALAT1 expression predicts a poor prognosis of cervical cancer and promotes cancer cell growth and invasion. Int J Clin Exp Pathol 19: 3187-3193, 2015. 